\title{
Surface Engineering of Gold Nanorods for Cytochrome $c$ Bioconjugation: An Effective Strategy To Preserve the Protein Structure
}

Tiziana Placido, ${ }^{\dagger}$ Lorenzo Tognaccini, ${ }^{\ddagger}$ Barry D. Howes, ${ }^{\ddagger}$ Alessandro Montrone, ${ }^{\S}$ Valentino Laquintana, Roberto Comparelli, ${ }^{\dagger}$ M. Lucia Curri, ${ }^{\dagger}$ Giulietta Smulevich, ${ }^{*}, \star \odot$ and Angela Agostiano ${ }^{*}, \dagger, \S$

${ }^{\dagger}$ CNR-IPCF Istituto per I Processi Chimico-Fisici, S.S. Bari, c/o Dip. Chimica, Via Orabona 4, 70126 Bari, Italy

${ }^{\ddagger}$ Dipartimento di Chimica "Ugo Schiff”, Università di Firenze, Via della Lastruccia 3-13, Sesto Fiorentino, 50019 Florence, Italy

${ }^{\S}$ Università degli Studi di Bari-Dip. di Chimica, Via Orabona 4, 70126 Bari, Italy

"Dipartimento di Farmacia-Scienze del Farmaco, Università degli Studi di Bari, Via Orabona 4, 70126 Bari, Italy

\section{Supporting Information}

\begin{abstract}
The surface of gold nanorods (Au NRs) has been appropriately engineered to achieve a suitable interface for bioconjugation with horse heart cytochrome $c$ (HCc). $\mathrm{HCc}$, an extensively studied and well-characterized protein, represents an ideal model for nanoparticle (NP)-protein conjugation studies because of its small size, high stability, and commercial availability. Here, the native state of the protein has been demonstrated for the first time, by means of Raman spectroscopy, to be retained upon conjugation with the anisotropic $\mathrm{Au}$ nanostructures, thus validating the proposed protocol as specifically suited to mostly preserve the plasmonic properties of the NRs and to retain the structure of the

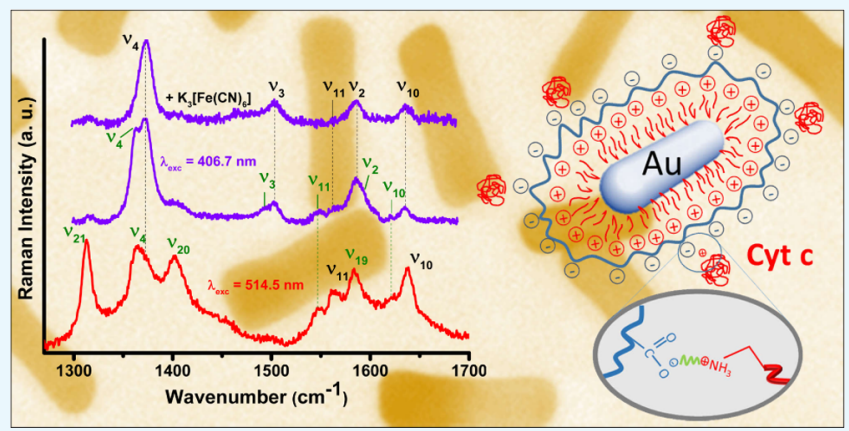
protein. The successful creation of such bioconjugates with the retention of the protein structure and function along with the preservation of the NP properties represents a challenging but essential task, as it provides the only way to access functional hybrid systems with potential applications in biotechnology, medicine, and catalysis. In this perspective, the organic capping surrounding the $\mathrm{Au}$ NRs plays a key role, as it represents the functional interface for the conjugation step. Cetyltrimethylammonium bromide-coated $\mathrm{Au}$ NRs, prepared by using a seed-mediated synthetic route, have been wrapped with polyacrylic acid (PAA) by means of electrostatic interactions following a layer-by-layer approach. The resulting waterdispersible negatively charged AuNRs@PAA NPs have then been electrostatically bound to the positively charged HCc. The bioconjugation procedure has been thoroughly monitored by the combined analysis of UV-vis absorption, resonance Raman and Fourier transform infrared spectroscopies, transmission electron microscopy microscopy, and $\zeta$-potential, which verified the successful conjugation of the protein to the nanorods.
\end{abstract}

\section{INTRODUCTION}

In the recent years, new perspectives have been offered by anisotropic gold nanoparticles (NPs) such as nanorods [gold nanorods ( $\mathrm{Au} \mathrm{NRs)]} \mathrm{because} \mathrm{of} \mathrm{their} \mathrm{interesting} \mathrm{optical}$ properties, which have been exploited especially in biomedical diagnostics, in photothermal therapy, and as dark field imaging contrast agents and surface enhanced Raman spectroscopic (SERS) substrates. ${ }^{1-8}$ Au NRs are characterized by two surface plasmon bands, namely, a transverse surface plasmon mode located in the visible region around $520 \mathrm{~nm}$ and a longitudinal surface plasmon mode at longer wavelengths, which can be tuned from the visible to the near-infrared (NIR) region by varying the aspect ratio (length-to-width ratio) of the $\mathrm{Au}$ NRs. ${ }^{9-11}$ Bioconjugates of Au NRs are exceptionally promising candidates for important biomedical applications, especially when the resonances of the NRs are tuned to the NIR, where biological tissues are relatively transparent. In particular, NPprotein hybrid systems have already demonstrated their potential for many applications in research and diagnostics, for example, optical biosensing, ${ }^{12}$ imaging of tissues, cells, or macromolecules, ${ }^{5,13,14}$ and physicochemical manipulation of biological systems. ${ }^{15}$ In this perspective, it is fundamental that the functional properties of the bioconjugates are maintained during the preparation. To accomplish this bioconjugation task, the nature of the NP interface and the adsorption are crucial, as they can determine conformational changes, denaturation, or undesirable protein orientations with respect to the substrate ${ }^{16}$ that endanger their activity. To overcome these problems, NPs

Received: April 13, 2018

Accepted: April 26, 2018

Published: May 7, 2018 


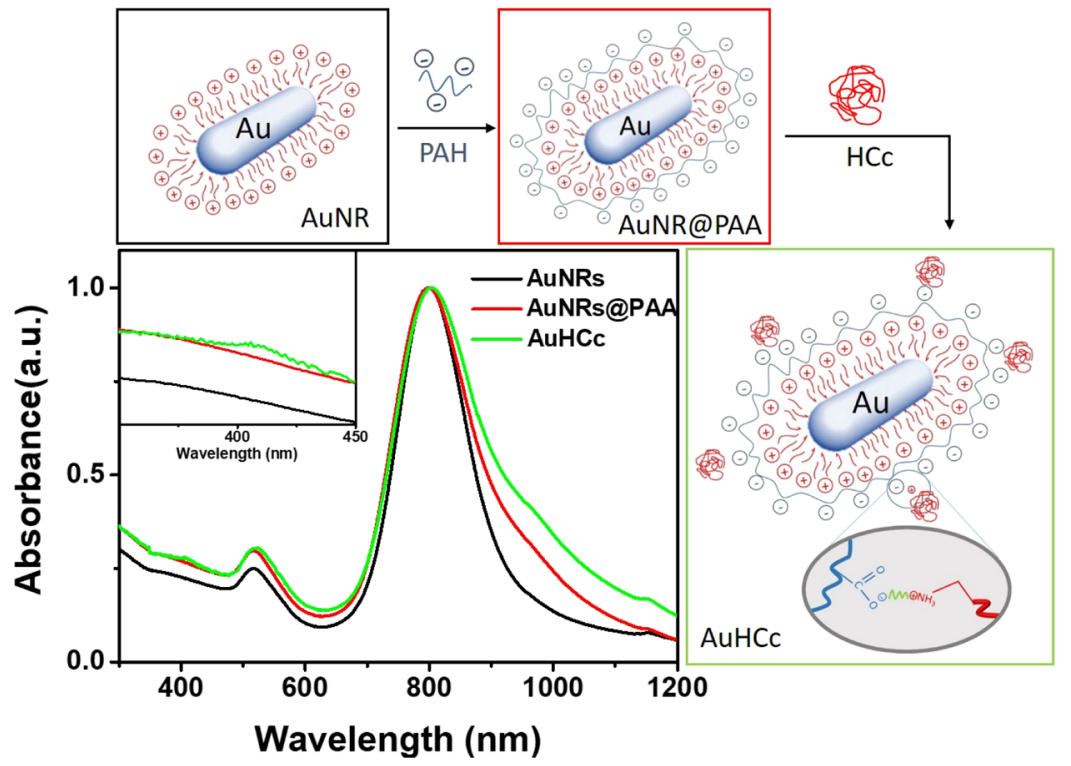

Figure 1. Evolution of normalized UV-vis absorption spectra of "as prepared" Au NRs (black line), Au NRs coordinated by PAA (red line), and HCc-conjugated Au NRs (AuHCc, green line). The inset shows a zoom of the spectra within the 350-450 nm wavelength range. The calculated $\mathrm{HCc}$ concentration of the AuHCc solution is about $10^{-6} \mathrm{M}$ (molar absorptivity $1.06 \mathrm{M}^{-1} \mathrm{~cm}^{-1}$ ). ${ }^{37}$ A sketch presenting the multiple functionalization process of $\mathrm{Au}$ NRs with $\mathrm{HCc}$ is also included.

have been modified with small molecules ${ }^{17}$ or polymeric matrixes ${ }^{18-20}$ and characterized by means of bioanalytical techniques involving transduction of protein-based signals for detection, including electrochemistry, ${ }^{21}$ and SERS ${ }^{22}$ to verify the native state of protein. The goal of this work is to bioconjugate water-dispersible Au NRs, coated with the positively charged surfactant stabilizer [cetyltrimethylammonium bromide (CTAB)], to horse heart cytochrome $c$ (HCc), while preserving the nanostructure geometry-dependent properties and, at the same time, the protein structure. Generally, three approaches have been proposed in the literature to link proteins to $\mathrm{Au}:^{23}$ (i) direct anchoring on the CTAB coating of Au NRs by exploiting electrostatic adsorption, provided that the protein is negatively charged; (ii) interposing a negatively charged layer as a "glue" between CTAB and the protein; $^{24}$ and (iii) using as intermediate linkers bifunctional molecules containing a functional group with strong or good affinity for the metal surface [i.e., cyano $(-\mathrm{CN})$, mercapto $(-\mathrm{SH})$, carboxylic acid $(-\mathrm{COOH})$, and amino $\left(-\mathrm{NH}_{2}\right)$ groups] to, at least partially, displace the native bilayer and, on the other side, a functional group that interacts with the protein by means of covalent or noncovalent bonds. ${ }^{23} \mathrm{HCc}$ is used here as a protein probe, being a small, single chain, mitochondrial protein that plays an important role both in electron transfer and in programmed cell death (apoptosis). The identification of a strategy suited to bioconjugate $\mathrm{HCc}$ on $\mathrm{Au}$ NRs can be of great interest for biological applications and can allow the refinement of new operating procedures for protein bioconjugation. A possible direct covalent interaction of horse heart $\mathrm{HCc}$ with the bare Au NRs surface is not viable because of the lack of functional groups able to covalently bind the Au surface. Furthermore, HCc exhibits a positively charged domain around the exposed heme edge ${ }^{25,26}$ that hampers any direct electrostatic interaction with the positively charged CTAB-capped Au NRs. In addition, a surface functionalization step to replace the pristine $\mathrm{CTAB}$ coating with bifunctional molecules more suited for subsequent binding of $\mathrm{HCc}$ could cause irreversible aggregation of $\mathrm{Au} \mathrm{NRs}$ or a continuous release of the residual $\mathrm{CTAB}$, thus endangering the protein stability also. ${ }^{27}$ A reliable strategy, typically used to modify the surface chemistry and simultaneously protect the native NR coating from possible detachment of CTAB molecules is based on polyelectrolyte wrapping, ${ }^{27}$ that endows the nanostructure surface with functional groups that enable their conjugation with biomolecules, including DNA, peptides, antibodies, and proteins by means of either electrostatic or covalent interactions. ${ }^{28}$ We herein investigate the viability of poly(allylamine hydrochloride) (PAA) which, being a weak polyelectrolyte, allows the interaction between $\mathrm{HCc}$ and $\mathrm{Au}$ $\mathrm{NRs}$ to be modulated by controlling ionic strength and $\mathrm{pH}$, thus obtaining a suitable wrap for the Au NRs.

Recent studies have shown that the conformation of $\mathrm{HCc}$ adsorbed on Au NPs depends on surface coverage and NP size. $^{29}$ To date, preservation of the native structure of HCc has been achieved on electrodes by using appropriate selfassembled monolayers, ${ }^{26,30}$ but bioconjugation of colloidal $\mathrm{Au}$ NPs has mostly resulted in protein denaturation. ${ }^{22,31-35}$ In particular, the conjugation of $\mathrm{HCc}$ to Au NPs has been found to preserve the HCc structure only when the structure has been decorated with aggregated $\mathrm{Ag}$ nanospheres. ${ }^{22}$ The bioconjugation of Au NRs with proteins is even more difficult. In fact, the functionalization of Au NRs becomes more crucial because of the geometrical features of the nanostructures, as NRs are particularly sensitive to self-assembly and aggregation problems ${ }^{36}$ and to the possible toxicity issues arising from the capping molecules that may detach from the nanostructures. ${ }^{27}$ Herein, "as prepared" CTAB-capped Au NRs have been functionalized by using the electrostatic layer-by-layer approach to proceed with their subsequent decoration with HCc. Namely, the NRs have been coated with a negatively charged polyelectrolyte, PAA, which effectively enables the interaction of the NRs with HCc, which is positively charged because of the presence of Lys residues in its structure. The bioconjugation of the resulting AuNRs@PAA NPs with HCc, thoroughly characterized by means of $\zeta$-potential, UV-vis absorption spectroscopy, transmission electron microscopy, 
Fourier transform infrared (FTIR) and resonance Raman (RR) spectroscopies, has demonstrated, for the first time, that the native state of the protein is preserved. The overall results indicate that the proposed protocol is specifically suited to accomplish an effective interface engineering of the Au NRs to bioconjugate $\mathrm{HCc}$, enabling the design of hybrid nanosystems with a precise control of the inorganic geometry-dependent properties and the final biological functionality.

\section{RESULTS AND DISCUSSION}

The characterization of the surface interactions between $\mathrm{Au}$ NRs and proteins is of importance for many potential applications. However, it has been proven that it is very difficult to achieve protein binding without damaging the native structure or modifying the NR plasmonic properties. Furthermore, neither of the two moieties have specific anchor points that facilitate covalent binding; therefore, the task represents a significant challenge. To overcome such difficulties and attain effective bioconjugation of the probe protein $\mathrm{HCc}$, the surface properties of the Au NRs have been modified by means of a layer-by-layer electrostatic functionalization procedure. Herein, CTAB-coated Au NRs with an aspect ratio of $4.2 \pm 0.4$ (diameter $=14.1 \pm 1.6 \mathrm{~nm}$; length $=58.5 \pm$ $5.8 \mathrm{~nm}$ ) have been synthesized by using the seed-mediated approach, which results in NRs that have the surface coated with a bilayer of CTAB molecules, which ensures a very good particle dispersion in water, thus minimizing the risk of aggregation phenomena. Au NRs were prepared by reducing the $\mathrm{Au}(\mathrm{III})$ precursor at room temperature to $\mathrm{Au}(0)$ in the presence of $\mathrm{CTAB}$ as a surfactant.

The UV-vis spectrum of colloidal Au NRs is reported in Figure 1 (black line) and shows the typical two plasmon bands located at 516 and $800 \mathrm{~nm}$, corresponding to transverse and longitudinal plasmon modes, respectively. Moreover, the longitudinal plasmon band is quite narrow and intense compared with the transverse band, suggesting a high yield of nearly monodisperse Au NRs.

Moreover, the ammonium-terminated polar heads of the surfactants interact with water molecules in the surrounding environment, conferring a positive charge to the Au NRs, as demonstrated by the $\zeta$-potential measurements (Table 1, Figure S1).

Table 1. Particle Size $(d)$, Size Distribution and Surface Charge of Au NRs

\begin{tabular}{lccc}
\multicolumn{1}{c}{ sample } & $\begin{array}{c}d \text { mean }(\mathrm{nm}) \\
z \text {-average }\end{array}$ & PDI $^{a}$ & $\begin{array}{c}\zeta \text {-potential } \\
(\mathrm{mV})\end{array}$ \\
Au NRs & $86.1 \pm 0.5$ & $0.223 \pm 0.046$ & $+36.4 \pm 1.0$ \\
AuNRs@PAA & $127.2 \pm 3.0$ & $0.203 \pm 0.071$ & $-37.9 \pm 0.3$ \\
AuHCc & $160.2 \pm 1.6$ & $0.115 \pm 0.032$ & $-18.4 \pm 1.0$
\end{tabular}

${ }^{a} \mathrm{PDI}=$ polydispersity index.

The positively charged quaternary ammonium head of the $\mathrm{CTAB}$ molecules coating the Au NRs promote the electrostatic binding of the positively charged HCc by interaction with the negatively charged PAA (see the sketch in Figure 1). Such a polyelectrolyte wrapping of the NRs is, in principle, able to limit the possible release of the $\mathrm{CTAB}$ molecules of the bilayer coating the NR surface, thus representing a safe "glue" compatible with the presence of biomolecules. The most investigated polyelectrolyte to electrostatically link $\mathrm{HCc}$ has been polystyrene sulfonated (PSS). However, the strong electrostatic interaction with the negative charges of PSS has been found to cause extensive disruption of the native structure of $\mathrm{HCc}^{26,38}$ Conversely, PAA, being a weak polyelectrolyte, allows the interaction between $\mathrm{HCc}$ and $\mathrm{Au} \mathrm{NRs}$ to be modulated by the tuning of ionic strength and $\mathrm{pH}$, thus obtaining the most-suited wrap for the Au NRs. In fact, this process has been demonstrated to be affected not only by the polymer charge density and the initial charge distribution on the CTAB-capped $\mathrm{Au}^{27}$ but also by the balance between polymeric unit-NP attraction and repulsion among polymeric units. $^{39}$ The charge density of PAA is a function of the solution $\mathrm{pH}$ and, therefore, affects the arrangement of the chains and their conformation upon surface adsorption. ${ }^{40}$ In fact, for $\mathrm{pH}>$ $\mathrm{p} K_{\mathrm{a}}\left(\mathrm{PAA}, \mathrm{p} K_{\mathrm{a}}=4.8\right)$, many of the PAA carboxylic groups are deprotonated, thus inducing stretching of the polymeric chains because of reciprocal electrostatic repulsion. ${ }^{41}$ In this study, PAA has been incubated in solution with the Au NRs at $\mathrm{pH}$ 7, obtaining a complete surface coverage. This is demonstrated by the inversion of the $\zeta$-potential value (Table 1 and Figure S1) from the initial positive charge of the Au NRs $(+36.4 \mathrm{mV})$ to the negative charge of AuNRs@PAA (-37.9 mV). The ionic strength of the PAA solution has been left unchanged although, in principle, tuning this parameter could help optimize the electrostatic screening of the polyelectrolyte in solution. Nevertheless, any addition of salt would be detrimental, as it is known to cause aggregation of Au NRs. ${ }^{42}$ As can be seen in the absorption spectrum of the AuNRs@PAA sample, after the purification procedure, the coverage with the PAA layer does not induce any significant change in the position of the longitudinal plasmon band (Figure 1, red line). At this stage, the AuNRs@PAA solution has been incubated with an excess of positively charged $\mathrm{HCc}$ for $1 \mathrm{~h}$ to promote electrostatic anchoring of the protein on the Au NRs (AuHCc sample). The unbound $\mathrm{HCc}$ has been removed by centrifugation cycles, to precipitate and isolate only functionalized Au NRs. The experimental conditions are decisive not only to control the amount of protein anchored on the Au NRs surface by simply varying the incubation time of the protein in the AuNRs@PAA solution but also to modulate the strength of the interaction between HCc and AuNRs@PAA, that may affect the extent of protein denaturation at the NR surface. ${ }^{31,43}$ In addition, it has been reported that a higher protein coverage could result in an overcrowded arrangement and thus enhance the proteinprotein and protein-NRs interactions that, consequently, may affect protein folding and, ultimately, its activity. ${ }^{44,45}$ Therefore, to maintain constant the number of free carboxylates of PAA coating the Au NRs, the AuNRs@PAA solution has been washed and redispersed in phosphate buffer solution at physiological $\mathrm{pH}(\mathrm{pH} 7)$. The presence of $\mathrm{HCc}$ in the $\mathrm{Au}$ NRs solution is clearly shown by the absorption spectrum of the AuHCc sample. The appearance of the Soret band of native $\mathrm{HCc}\left(\mathrm{Fe}^{3+}\right)$ at $409 \mathrm{~nm}$, concomitant with the slight red shift of the longitudinal plasmon band because of modification of the chemical environment, is clearly evident (Figure 1, green line). Remarkably, because of its low molecular weight, the free $\mathrm{HCc}$ in solution does not precipitate along with the bioconjugated $\mathrm{Au}$ NRs upon numerous washing cycles of the sample by centrifugation. In fact, no precipitate is observed when pure $\mathrm{HCc}$ solution was centrifugated in the same experimental conditions as the bioconjugated sample. The shoulder on the longitudinal plasmon band observed at higher wavelengths can be ascribed to minor aggregation phenomena arising from the purification/centrifugation cycles. 


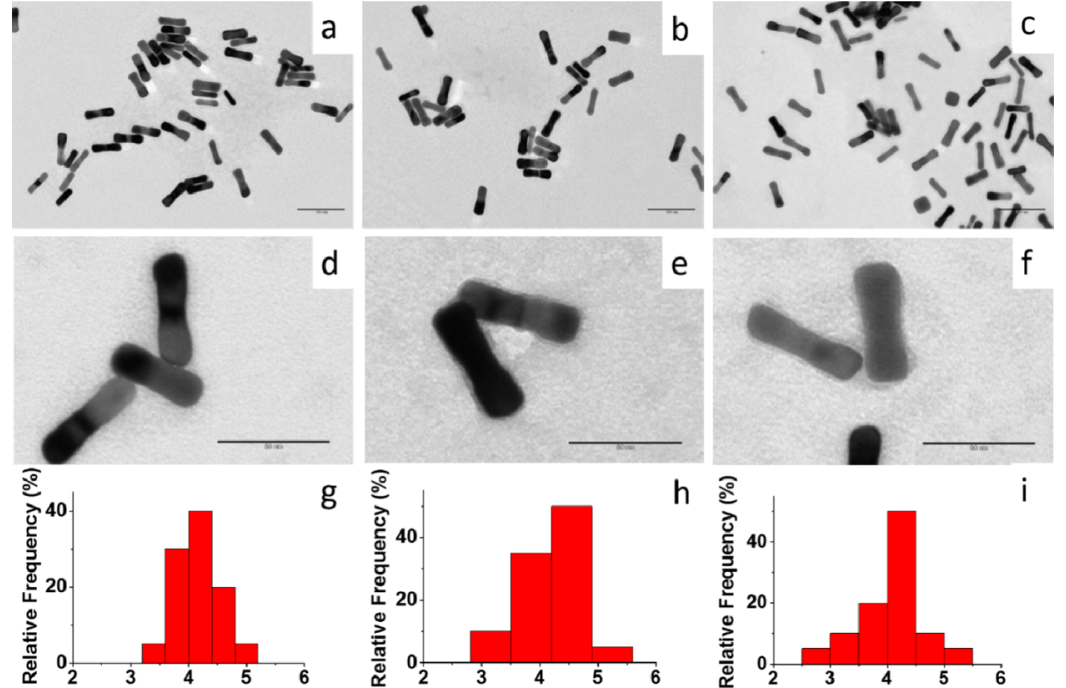

Figure 2. TEM images of as-synthesized Au NRs (a), Au NRs coordinated by PAA (b) and HCc-conjugated Au NRs (c) (100 nm scale bar). The TEM images of the same samples captured in negative staining mode are shown in $(\mathrm{d}-\mathrm{f})$. (50 nm scale bar). The corresponding aspect ratio statistical analyses of the particles are presented in $(\mathrm{g}-\mathrm{i})$.

The $\zeta$-potential value after incubation with HCc (Table 1 , Figure S1) decreases because of the reduction in the number of free PAA carboxyl groups, which confirms that the protein is well-anchored and also indicates a good stability of the prepared NRs. Transmission electron microscopy (TEM) images of as-synthesized Au NRs (a), Au NRs coordinated by PAA (b), and HCc-conjugated Au NRs are reported in Figure 2. The TEM micrograph in Figure 2a shows that the shape and size distribution of the Au NRs is quite uniform with an aspect ratio of $4.2 \pm 0.4$ (Figure $2 \mathrm{~g}$ ). The TEM analysis of the aspect ratios of the particles for AuNRs@PAA and AuHCc (Figure $2 \mathrm{~h}, \mathrm{i}$ ), performed on the TEM images (Figure $2 \mathrm{~b}, \mathrm{c}$ ), shows that they are $4.2 \pm 0.5$ and $4.1 \pm 0.5$, respectively. This confirms that the $\mathrm{Au}$ NRs preserve their size and shape after the polyelectrolyte wrapping and HCc binding steps. Therefore, the slight modification of the longitudinal plasmon band position observed in Figure 1 can be safely ascribed to the modification of the chemical environment of Au NRs. Interestingly, TEM images of the negatively stained samples (Figure $2 \mathrm{~d}-\mathrm{f}$ ) highlight the modification of the Au NRs organic coating, that passes from a uniform and thin layer for Au NRs to an inhomogeneous coating of about 2.5 nm for AuNRs@PAA, consistent with the less compact structure of a polyelectrolyte layer. Such features are even more evident when the HCc is anchored to the Au NRs. Furthermore, the diameter analysis by dynamic light scattering (DLS) (Table 1, Figure S2) shows that the $\mathrm{Au}$ NRs are characterized by an equivalent spherical shape and rather homogeneous in size: $86.1 \pm 0.5 \mathrm{~nm}$ for Au NRs, $127.2 \pm 3.0 \mathrm{~nm}$ for AuNRs@PAA, and 160.2 $\pm 1.6 \mathrm{~nm}$ for $\mathrm{AuHCc}$, whereas their diameters are higher than those observed by TEM. This discrepancy can be explained by taking into account that DLS probes the hydrodynamic diameter, which includes the hydration shell due to the PAA coating. In addition, the DLS analysis of anisotropic particles, such as Au NRs, has to be interpreted cautiously because of the possible occurrence of multiple diffusion modes and must be considered only qualitatively. Nevertheless, such values are reliable enough to provide a useful indication of the size evolution trend of the nanostructure along the overall functionalization process. The diffusion of rod-like NPs is more complicated to describe than that of nanospheres because the presence of two geometrical parameters (length $l$ and radius $R$ ) requires that both the rotational and the translational diffusion coefficients be taken into account for the treatment of the rod-like particle hydrodynamics. In addition, the overall translational diffusion coefficient is determined by a suitable combination of the diffusion coefficients for motion parallel $\left(D_{\|}\right)$and perpendicular $\left(D_{\perp}\right)$ to the long axis of the $\mathrm{NR}^{46,47}$

The bioconjugation process has also been monitored by FTIR investigation (Figure 3, Table S1). The intense signal at $1702 \mathrm{~cm}^{-1}$ and the broad band with medium intensity at 1248 $\mathrm{cm}^{-1}$ are typically associated with $\nu(\mathrm{C}=\mathrm{O})$ and $\nu(\mathrm{C}-\mathrm{O})$ stretching modes of carboxylic group dimers. The weak bending $\delta(\mathrm{O}-\mathrm{H})$ mode at $814 \mathrm{~cm}^{-1}$ further confirms the presence of carboxylic group dimers in PAA. In addition, the asymmetric stretching $\nu(\mathrm{C}-\mathrm{O})$ mode, associated with COO- at $1558 \mathrm{~cm}^{-1}$, is coupled with the weaker symmetric $\nu(\mathrm{C}-\mathrm{O})$ mode at $1408 \mathrm{~cm}^{-1}$, indicating the presence of carboxylate ions. ${ }^{48}$ Therefore, this suggests that at the $\mathrm{pH}$ used ( $\mathrm{pH} 7)$, the carboxylic groups of the PAA stock solution are not completely deprotonated and thus both dimers and carboxylate ions coexist. Remarkably, when PAA interacts with CTAB to wrap the $\mathrm{Au} \mathrm{NRs}$, the intense band at $1702 \mathrm{~cm}^{-1}$ significantly decreases, leaving the intense band at $1558 \mathrm{~cm}^{-1}$ of the carboxylate ions, which interacts with the ammonium heads of $\mathrm{CTAB}$ (Figure 3B). The binding of $\mathrm{HCc}$ to the PAA layer results in the appearance of the typical band at $1652 \mathrm{~cm}^{-1}$ (Figure 3D), assigned to the $\nu(\mathrm{C}=\mathrm{O})$ stretching mode of amide $\mathrm{I}$ of the protein (Figure $3 \mathrm{C}$ ) that is a very sensitive probe of protein linkages. ${ }^{35}$ In fact, the intensity of this signal slightly decreases when $\mathrm{HCc}$ electrostatically interacts with PAA.

Therefore, the overlap of the HCc and PAA bands in the 1500-1700 $\mathrm{cm}^{-1}$ region does not permit any further considerations on the bioconjugation event. However, any denaturation of the protein can be ruled out, as indicated by the lack of a band at $1635 \mathrm{~cm}^{-1}$ in the FTIR spectrum of AuHCc, typically ascribed to denatured HCc (Figure 3D). ${ }^{49}$

The AuHCc RR spectra (Figure 4, red) show, for both 406.7 (b) and 514.5 (a) nm excitation, the coexistence of the oxidized and reduced $\mathrm{HCc}$ forms. For $406.7 \mathrm{~nm}$ excitation, in resonance 


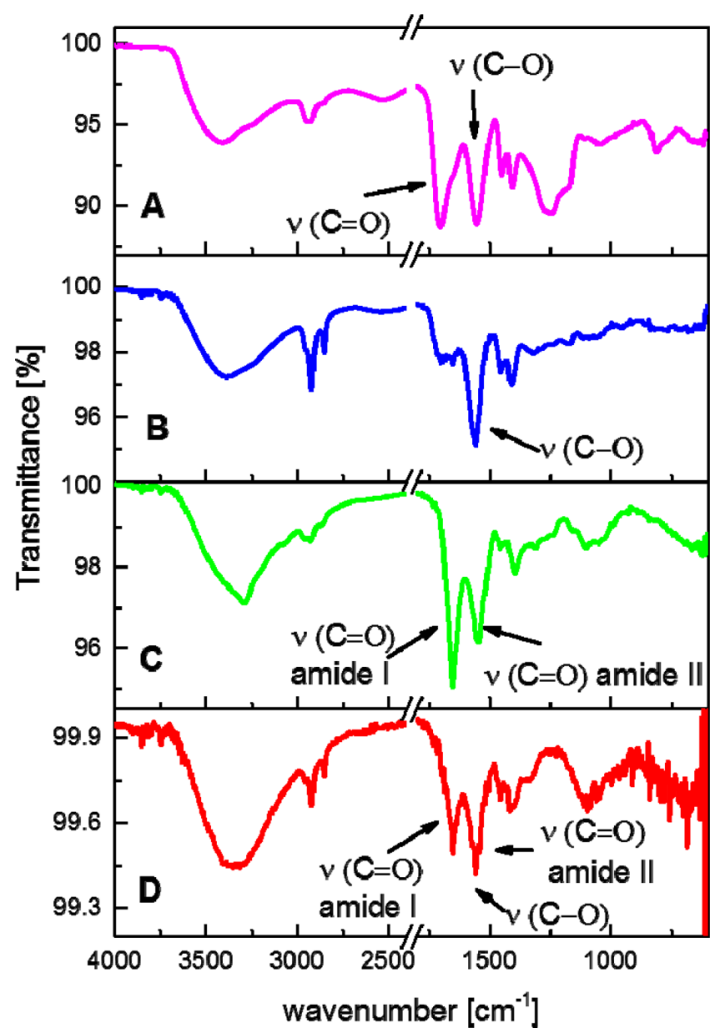

Figure 3. ATR-FTIR spectra of PAA (A), AuNRs@PAA (B), HCc (C), and $\mathrm{AuHCc}(\mathrm{D})$.

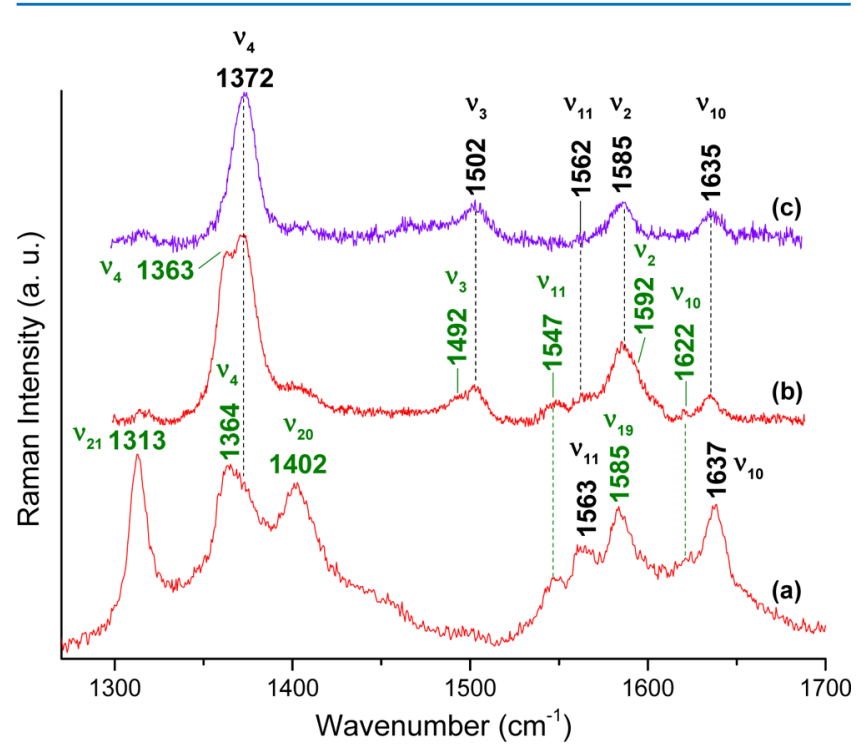

Figure 4. RR spectra of AuHCc (red) and AuHCc after addition of $\mathrm{K}_{3}\left[\mathrm{Fe}(\mathrm{CN})_{6}\right]$ (violet) obtained with the 514.5 (a) and $406.7(\mathrm{~b}, \mathrm{c}) \mathrm{nm}$ excitation wavelengths. The bands assigned to $\left(\mathrm{Fe}^{2+}\right)$ and $\left(\mathrm{Fe}^{3+}\right) \mathrm{HCc}$ are indicated in green and black, respectively. Experimental conditions: laser power at the sample 40 (a) and $10 \mathrm{~mW}(\mathrm{~b}, \mathrm{c})$; average of 15 spectra with 2 h 30 min integration time (a), 4 spectra with 40 min integration time (b), and 8 spectra with $80 \mathrm{~min}$ integration time (c). The spectra have been shifted along the ordinate axis to allow better visualization.

with the HCc Soret bands of both the ferric $(409 \mathrm{~nm})$ and ferrous $(417 \mathrm{~nm})$ forms, the spectra are dominated by the totally symmetric modes of native $\mathrm{HCc}$. In fact, for the $\mathrm{Fe}^{2+}$ form, $\nu_{4}, \nu_{3}, \nu_{2}$, and $\nu_{10}$ bands are observed at 1363, 1492, 1592, and $1622 \mathrm{~cm}^{-1}$, and for the $\mathrm{Fe}^{3+}$ form, $\nu_{4}, \nu_{3}, \nu_{2}$, and $\nu_{10}$ bands are observed at $1372,1502,1585$, and $1637 \mathrm{~cm}^{-1}$, respectively. The full assignment is reported in Table S2. Complete HCc oxidation has been achieved by adding a minimum amount of $\mathrm{K}_{3}\left[\mathrm{Fe}(\mathrm{CN})_{6}\right]$ solution to the AuHCc solution (Figure 4, violet), without any change in the position of the Au plasmon bands and the HCc Soret band (data not shown). This suggests that the reduced species represents only a small amount of the total HCc bound to the NRs, even though it is clearly visible in the Raman spectrum. This apparent discrepancy probably results from different resonance intensification factors and cross sections of the core-size marker bands of the reduced form compared with the oxidized species. Moreover, the treatment with $\mathrm{K}_{3}\left[\mathrm{Fe}(\mathrm{CN})_{6}\right]$ did not induce any detachment of the protein from the NRs, as verified by subsequent centrifugation and washing of the sample (Figure S5). Nevertheless, after the washing, the reduced species appears again in the Raman spectrum. Upon $514.5 \mathrm{~nm}$ excitation (Figure 4, a), in resonance with the $\mathrm{Q}$ band transitions of $\mathrm{HCc}(\beta$-band at 530 and $520 \mathrm{~nm}$ for the ferric and ferrous forms, respectively), the spectrum is characterized, as expected, by $\mathrm{B}_{1 g}, \mathrm{~A}_{2 \mathrm{~g}}$, and $\mathrm{B}_{2 \mathrm{~g}}$ modes. The bands at 1313,1402 , and $1585 \mathrm{~cm}^{-1}$ are assigned to $\nu_{21}, \nu_{20}$, and $\nu_{19}$ of the ferrous form, respectively. However, the coexistence of the reduced and oxidized forms causes some changes in the relative intensity ratios of the bands compared with the spectra of the pure species ${ }^{50,51}$ (Table S2).

The HCc adsorbed on the NRs retains its native conformation (i.e., the heme iron is bound to the $\mathrm{His}_{18}$ and Met $_{80}$ internal ligands), as demonstrated by the close correspondence of the difference spectra obtained by subtracting alternatively the native $\mathrm{HCc}\left(\mathrm{Fe}^{2+}\right)$ (Figure 5, bc) and the native $\mathrm{HCc}\left(\mathrm{Fe}^{3+}\right)$ (Figure 5, b-a) from the spectrum

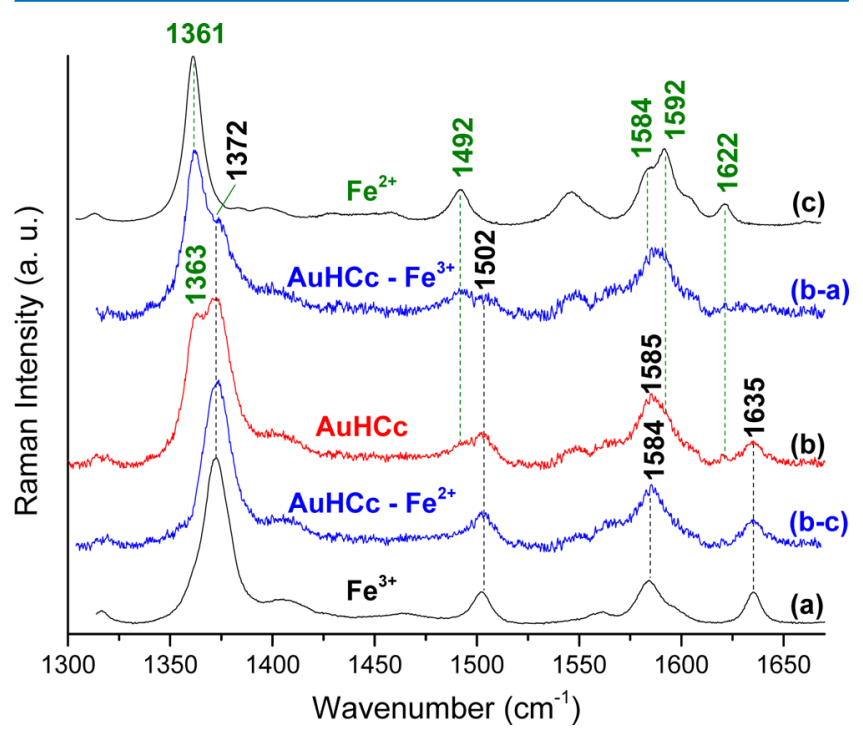

Figure 5. RR spectrum of $A u H C c(b)$ together with the difference spectra (b-c, b-a) after subtraction of the pure ferric $\mathrm{HCc}(\mathrm{a})$ and ferrous HCc (c) solution spectra. Experimental conditions: (a) 406.7 $\mathrm{nm}$ excitation wavelength, laser power at the sample $5 \mathrm{~mW}$, and average of 5 spectra with 25 min integration time; (b) $406.7 \mathrm{~nm}$ excitation wavelength, laser power at the sample $10 \mathrm{~mW}$, and average of 48 spectra with $4 \mathrm{~h}$ integration time; and (c) $413.1 \mathrm{~nm}$ excitation wavelength, laser power at the sample $5 \mathrm{~mW}$, and average of 4 spectra with 20 min integration time. The intensities are normalized to that of the $\nu_{4}$ band. The spectra have been shifted along the ordinate axis to allow better visualization. 
of AuHCc (Figure 5b). In fact, the core-size marker band frequencies are identical to those of the native protein in solution, indicating that no changes in the heme distortion, and hence ligation, ${ }^{52,53}$ have been induced by bioconjugation. This conclusion is confirmed by the "fingerprint" region between 300 and $450 \mathrm{~cm}^{-1}$ that shows that features are typical of a $\mathrm{Met}-\mathrm{Fe}-\mathrm{His}$ species (Figure 6). Indeed, previous reports

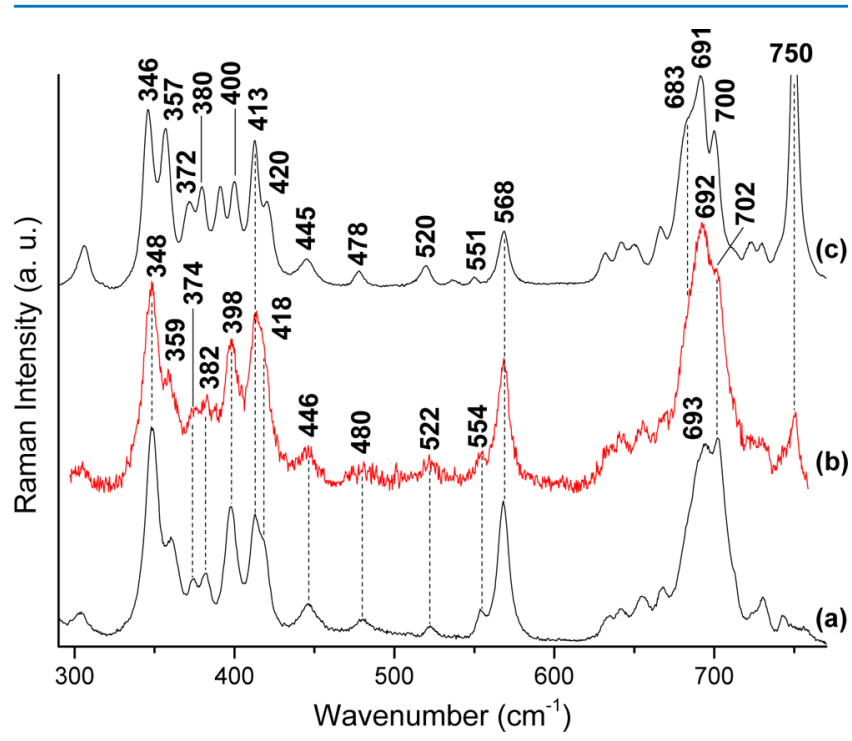

Figure 6. RR spectra of AuHCc (b, red) compared with ferric (a) and ferrous (c) HCc in the low-frequency region, obtained with the 406.7 $\mathrm{nm}$ excitation wavelength. Experimental conditions: laser power at the sample $5 \mathrm{~mW}(\mathrm{a}, \mathrm{c})$ and $10 \mathrm{~mW}(\mathrm{~b})$; average of 5 spectra with $25 \mathrm{~min}$ integration time (a), 18 spectra with $3 \mathrm{~h}$ integration time (b), and 4 spectra with $20 \mathrm{~min}$ integration time (c). The spectra have been shifted along the ordinate axis to allow better visualization.

found that upon denaturation the disruption of the $\mathrm{Met}_{80}$-iron bond occurs concomitantly with the loss of the secondary structure and the increase in the molecular size under equilibrium unfolding conditions. ${ }^{54}$ The replacement of the Met $_{80}$ heme ligand with other residues should cause marked changes both in the positions and in the relative intensities of the Raman bands. In particular, specific iron-ligand stretching vibrations have been identified for $\mathrm{His}_{18}-\mathrm{Fe}-\mathrm{Lys}$ and $\mathrm{His}_{18}-$ $\mathrm{Fe}-\mathrm{His}$ misligation as well as specific band patterns for $\mathrm{His}_{18}-$ $\mathrm{Fe}-\mathrm{OH}^{-}$and $\mathrm{His}_{18}-\mathrm{Fe}-\mathrm{H}_{2} \mathrm{O}$ conformations. ${ }^{55-61}$ Because the band frequencies and the intensity pattern of AuHCc (Figure $6 \mathrm{~b}$, red) are identical to those of the oxidized protein in solution (Figure 6a), with some minor changes due to the presence of a small percentage of the reduced species (Figure $6 \mathrm{~b})$, the HCc structure and heme ligation are fully conserved on the Au NR surface.

No SE(R)RS effect has been observed for both 406.7 (Figure S6) and $514.5 \mathrm{~nm}$ excitation because the intensity of the $\mathrm{HCc}$ signals is the same (or marginally lower) as that of a pure $\mathrm{HCc}$ buffer solution at $10^{-6} \mathrm{M}$ (Figure S3), that is, the calculated $\mathrm{HCc}$ concentration from the spectrum in Figure 1 (green line). Excitation with the 632.8, 647.1, 785, and $1064 \mathrm{~nm}$ laser lines has been also checked. However, no HCc signals have been observed (data not shown).

\section{CONCLUSIONS}

In conclusion, this work has demonstrated that waterdispersible CTAB-coated Au NRs, prepared by means of a seed-mediated synthetic route, can be wrapped with polyacrylic acid (PAA) by exploiting the electrostatic interactions between CTAB and PAA, without modifying the NRs optical properties. This layer-by-layer preparation scheme has been then used also to accomplish the electrostatic binding of positively charged $\mathrm{HCc}$ on the negatively charged AuNRs@PAA NPs. The thorough characterization of the bioconjugation procedure has established that the native state of the protein has been retained upon conjugation with $\mathrm{Au} \mathrm{NRs}$ and that the plasmonic properties of the $\mathrm{Au}$ NRs have also been mostly maintained. This result, confirming the success of the proposed protocol, represents the first example of $\mathrm{HCc}$ bioconjugation with anisotropic Au NPs. Hence, it is now possible to envisage the design of similar systems for biomimetic applications and for integration in bioelectronics devices.

\section{EXPERIMENTAL SECTION}

Materials. Sodium borohydride $\left(\mathrm{NaBH}_{4}, \sim 99 \%\right)$, L-ascorbic acid $(99 \%)$, potassium ferricyanide $\left(\mathrm{K}_{3}\left[\mathrm{Fe}(\mathrm{CN})_{6}\right]\right.$, analytical grade), sodium dihydrogen phosphate $\left(\mathrm{NaH}_{2} \mathrm{PO}_{4}\right.$, analytical grade), sodium hydrogen phosphate $\left(\mathrm{Na}_{2} \mathrm{HPO}_{4}\right.$, reagent grade), hydrogen tetrachloroaurate(III) trihydrate ( $\mathrm{HAuCl}_{4}$. $\left.3 \mathrm{H}_{2} \mathrm{O}, 99.9 \%\right)$, silver nitrate $\left(\mathrm{AgNO}_{3}, 99.9999 \%\right)$, PAA $\left(M_{\mathrm{n}} \approx\right.$ $130 \mathrm{kDa})$, potassium phosphate monobasic $(\geq 98 \%)$, potassium phosphate dibasic $(\geq 98 \%)$, potassium chloride $(\geq 99 \%)$, and HCc $\geq 95 \%$ based on mol. wt. 12 384) were purchased from Sigma-Aldrich. Stock solutions were prepared using deionized water (Millipore Milli-Q gradient A-10 system).

General Protocol for the "Seed-Mediated" Synthesis of Au Nanorods. CTAB-capped Au NRs with a longitudinal plasmon band at $800 \mathrm{~nm}$ were prepared using a suitably modified literature procedure commonly known as the seedmediated growth method. ${ }^{62,63}$ Briefly, a "seed" solution containing CTAB-stabilized Au NPs $(<3 \mathrm{~nm})$ was prepared by reducing the $\mathrm{Au}$ precursor, $\mathrm{HAuCl}_{4} \cdot 3 \mathrm{H}_{2} \mathrm{O}\left(5 \mathrm{~mL}, 5 \times 10^{-4}\right.$ $\mathrm{M})$, with $\mathrm{NaBH}_{4}(0.01 \mathrm{M})$ in CTAB $(5 \mathrm{~mL}, 0.2 \mathrm{M})$ and then was kept under vigorous stirring for $2 \mathrm{~h}$. At the same time, the $\mathrm{Au}$ NR growth solution was prepared: $8.5 \mathrm{mg}$ of $\mathrm{AgNO}_{3}, 18.22$ $\mathrm{g}$ of $\mathrm{CTAB}$, and $100 \mathrm{mg}$ of $\mathrm{HAuCl}_{4} \cdot 3 \mathrm{H}_{2} \mathrm{O}$ were dissolved in $500 \mathrm{~mL}$ of deionized water (CTAB dissolution was assisted by sonication). This mixture was kept at room temperature under continuous stirring. After $3 \mathrm{~min}, 7 \mathrm{~mL}$ of a $0.0778 \mathrm{M}$ aqueous solution of ascorbic acid was added dropwise to the above mixture. ${ }^{62}$ The mixture became colorless (indicative of the reduction of $\mathrm{Au}(\mathrm{III})$ to $\mathrm{Au}(\mathrm{I}),{ }^{64}$ and then, $0.8 \mathrm{~mL}$ of the "seed" solution was added to the entire growth solution. A dark-red color developed within $10 \mathrm{~min}$. After preparation, cycles of centrifugation were performed at $7000 \mathrm{rpm}$ for $20 \mathrm{~min}$ to wash the Au NRs eliminating uncoordinated CTAB.

Functionalization of Au NRs with HCc. The Au NRs were functionalized with $\mathrm{HCc}$ by exploiting electrostatic interactions in a layer-by-layer fashion (see the sketch in Figure 1). In the first step, the "as prepared" Au NRs were coated with PAA by incubation of $1 \mathrm{~mL}$ of Au NRs $\left(2 \times 10^{-9}\right.$ $\mathrm{M})$ and $1 \mathrm{~mL}$ of PAA $(1.6 \mathrm{mg} / \mathrm{mL}, \mathrm{pH} 7)$ for 20 min under gentle stirring. Then, this mixture was centrifuged two times at $10000 \mathrm{rpm}$ for $10 \mathrm{~min}$ to wash the Au NRs and eliminate free PAA. The AuNRs@PAA that precipitated upon centrifugation were dispersed in a phosphate buffer $(5 \mathrm{mM}, \mathrm{pH} 7)$ to ensure deprotonation of the PAA carboxylate groups and confer negative charges to the NR surface. Thus, in the second step, $200 \mu \mathrm{L}$ of the HCc solution $(0.5 \mathrm{mg} / \mathrm{mL})$ in $5 \mathrm{mM}$ phosphate buffer ( $\mathrm{pH}$ 7.0) was added to the as prepared AuNRs@PAA 
solution $\left(5 \times 10^{-10} \mathrm{M}\right)$, and this mixture was kept under gentle stirring for $1 \mathrm{~h}$. Finally, the obtained HCc-conjugated Au NRs (AuHCc) solution was centrifuged at $7000 \mathrm{rpm}$ for $10 \mathrm{~min}$, and washed with phosphate buffer $(5 \mathrm{mM}, \mathrm{pH} 7)$ to remove free $\mathrm{HCc}$ until no further decrease of the Soret band absorbance was observed. To ensure complete oxidization of the $\mathrm{HCc}$ adsorbed on the NRs, $2 \mu \mathrm{L}$ of a $\mathrm{K}_{3}\left[\mathrm{Fe}(\mathrm{CN})_{6}\right]$ solution ( 1 grain in $1 \mathrm{~mL}$ ) was added. The resulting solution was characterized by optical and morphological measurements to monitor the functionalization process.

Sample Characterization. UV-Visible Spectroscopy. UV-vis absorption spectra of solutions of the as prepared NRs and layer-by-layer multilayer-modified substrate were recorded at room temperature using a Varian Cary 5000 UVvis-NIR scanning spectrophotometer in the 300-1200 nm range or a Cary 60 (Agilent Technologies) with a $1 \mathrm{~mm}$ cuvette and a $600 \mathrm{~nm} / \mathrm{min}$ scan rate. Absorption spectra using a $5 \mathrm{~mm}$ NMR tube with a $300 \mathrm{~nm} / \mathrm{min}$ scan rate were measured both prior to and after $R R$ measurements to ensure that no degradation had taken place under the experimental conditions used. The $\mathrm{HCc}$ concentration was determined using the molar absorptivity $(\varepsilon)$ of $106 \mathrm{mM}^{-1} \mathrm{~cm}^{-1}$ at $409 \mathrm{~nm} .^{37}$

IR Spectroscopy. Mid-infrared spectra were acquired with a PerkinElmer Spectrum One FTIR spectrometer equipped with a deuteratedtryglicinesulfate detector. The spectral resolution used for all experiments was $4 \mathrm{~cm}^{-1}$. For attenuated total reflection (ATR) measurements, the internal reflection element (IRE) used was a three-bounce $4 \mathrm{~mm}$ diameter diamond microprism. Cast films were prepared directly on the IRE by depositing the solution of interest $(3-5 \mu \mathrm{L})$ onto the upper face of the diamond crystal and allowing the solvent to evaporate.

Transmission Electron Microscopy. TEM analyses were performed with a JEOL JEM-1011 microscope operating at 100 $\mathrm{kV}$. The specimens were prepared by depositing a few drops of aqueous NP dispersions onto a carbon-coated copper grid and then allowing the solvent to evaporate. The samples were stained with a $2 \%(\mathrm{v} / \mathrm{v})$ phosphotungstic acid solution for $30 \mathrm{~s}$. A statistical analysis of NR size distributions was performed on the basis of low-magnification TEM images with the help of ImageJ software. At least $150 \mathrm{NPs}$ were counted for each sample.

Particle Size, Size Distribution, and $\zeta$-Potential Measurements. The average hydrodynamic diameters ( $z$-average), size distribution (polydispersity index, PDI), and $\zeta$-potential were determined using Zetasizer Nano ZS, Malvern Instruments Ltd., Worcestershire, UK. Size and size distribution were measured by DLS after suspension in demineralized water at a suitable concentration $(\approx 0.1 \%$, w/v NRs concentration $)$. The ZP was determined by laser Doppler velocimetry after a suitable dilution of the samples with $\mathrm{KCl} 1 \mathrm{mM}(\approx 0.01 \%$, w/v NRs concentration).

$R R$ Measurements. $\mathrm{RR}$ spectra were recorded using a $5 \mathrm{~mm}$ NMR tube and $406.7\left(\mathrm{Kr}^{+}\right.$laser, Innova $300 \mathrm{C}$, Coherent, Santa Clara, CA) and $514.5 \mathrm{~nm}\left(\mathrm{Ar}^{+}\right.$laser, Innova 90 Coherent, Santa Clara, CA) excitation wavelengths. Backscattered light from a slowly rotating NMR tube was collected and focused into a triple spectrometer (consisting of two Acton Research SpectraPro 2300i and a SpectraPro 2500i in the final stage with a grating of 3600 or 1800 grooves $/ \mathrm{mm}$ ) working in the subtractive mode, equipped with a liquid nitrogen-cooled charge-coupled device detector. A spectral resolution of 1.2 $\mathrm{cm}^{-1}$ and spectral dispersion of $0.4 \mathrm{~cm}^{-1} /$ pixel were calculated theoretically on the basis of the optical properties of the spectrometer for the 3600 grating; for the 1800 grating, used to collect the RR spectra with the $514.5 \mathrm{~nm}$ excitation wavelength, the spectral resolution was $4 \mathrm{~cm}^{-1}$ and spectral dispersion 1.2 $\mathrm{cm}^{-1} /$ pixel.

The RR spectra were calibrated with indene and carbon tetrachloride as standards to an accuracy of $1 \mathrm{~cm}^{-1}$ for intense isolated bands. All the RR measurements were repeated several times under the same conditions to ensure reproducibility. To improve the signal-to-noise ratio, a number of spectra were accumulated and summed only if no spectral differences were noted. Because the spectra of the HCc-conjugated Au NRs $(\mathrm{AuHCc})$ solutions were characterized by a strong fluorescence background and a broad intense water band in the region 1550-1700 $\mathrm{cm}^{-1}$ on which the weak HCc signals were observed, for both excitation at 406.7 and $514.5 \mathrm{~nm}$, a blank sample spectrum (AuNRs@PAA in buffer solution, see Figure S7) was subtracted from all spectra. All spectra were baselinecorrected.

\section{ASSOCIATED CONTENT}

\section{S Supporting Information}

The Supporting Information is available free of charge on the ACS Publications website at DOI: 10.1021/acsomega.8b00719.

RR spectra of AuHCc obtained with 406.7 and $514.5 \mathrm{~nm}$ excitation wavelengths; RR spectra with $514.5 \mathrm{~nm}$ excitation wavelength of AuHCc compared with the spectra of pure $\left(\mathrm{Fe}^{3+}\right)$ and $\left(\mathrm{Fe}^{2+}\right)$ Cyt $c$ solutions at the same concentration calculated from the electronic absorption spectra of AuHCc; zetapotential distribution and size distribution by intensity recorded for $\mathrm{Au} \mathrm{NR}$, AuNRs@PCC, and AuHCc; Additional absorbance spectra of AuHCc; and tables of the assignments of the intense bands of the IR spectra and RR frequencies and vibrational assignments of Cyt $c$ and AuHCC (PDF)

\section{AUTHOR INFORMATION}

\section{Corresponding Authors}

*E-mail: giulietta.smulevich@unifi.it (G.S.).

*E-mail: angela.agostiano@uniba.it (A.A.).

ORCID

Roberto Comparelli: 0000-0003-4640-7204

Giulietta Smulevich: 0000-0003-3021-8919

\section{Author Contributions}

T.P. and L.T. contributed equally. The manuscript was written through contributions of all authors. All authors have given approval to the final version of the manuscript.

\section{Notes}

The authors declare no competing financial interest.

\section{ACKNOWLEDGMENTS}

The work has been supported by the Italian National PRIN 2010-2011 (no. 2010C4R8M8) and NANOMAX-integrable sensors for pathological biomarkers diagnosis (N-CHEM) projects.

\section{REFERENCES}

(1) Alkilany, A. M.; Thompson, L. B.; Boulos, S. P.; Sisco, P. N.; Murphy, C. J. Gold nanorods: Their potential for photothermal therapeutics and drug delivery, tempered by the complexity of their biological interactions. Adv. Drug Delivery Rev. 2012, 64, 190-199. 
(2) Jain, P. K.; Huang, X.; El-Sayed, I. H.; El-Sayed, M. A. Noble Metals on the Nanoscale: Optical and Photothermal Properties and Some Applications in Imaging, Sensing, Biology, and Medicine. Acc. Chem. Res. 2008, 41, 1578-1586.

(3) Kuo, W.-S.; Chang, C.-N.; Chang, Y.-T.; Yang, M.-H.; Chien, Y.H.; Chen, S.-J.; Yeh, C.-S. Gold Nanorods in Photodynamic Therapy, as Hyperthermia Agents, and in Near-Infrared Optical Imaging. Angew. Chem., Int. Ed. 2010, 49, 2711-2715.

(4) Ros, I.; Placido, T.; Amendola, V.; Marinzi, C.; Manfredi, N.; Comparelli, R.; Striccoli, M.; Agostiano, A.; Abbotto, A.; Pedron, D.; Pilot, R.; Bozio, R. SERS Properties of Gold Nanorods at Resonance with Molecular, Transverse, and Longitudinal Plasmon Excitations. Plasmonics 2014, 9, 581-593.

(5) Daniel, M.-C.; Astruc, D. Gold Nanoparticles: Assembly, Supramolecular Chemistry, Quantum-Size-Related Properties, and Applications toward Biology, Catalysis, and Nanotechnology. Chem. Rev. 2004, 104, 293-346.

(6) Burda, C.; Chen, X.; Narayanan, R.; El-Sayed, M. A. Chemistry and Properties of Nanocrystals of Different Shapes. Chem. Rev. 2005, $105,1025-1102$.

(7) Lee, K.-S.; El-Sayed, M. A. Gold and Silver Nanoparticles in Sensing and Imaging: Sensitivity of Plasmon Response to Size, Shape, and Metal Composition. J. Phys. Chem. B 2006, 110, 19220-19225.

(8) Njoki, P. N.; Lim, I.-I. S.; Mott, D.; Park, H.-Y.; Khan, B.; Mishra, S.; Sujakumar, R.; Luo, J.; Zhong, C.-J. Size Correlation of Optical and Spectroscopic Properties for Gold Nanoparticles. J. Phys. Chem. C 2007, 111, 14664-14669.

(9) Pérez-Juste, J.; Pastoriza-Santos, I.; Liz-Marzán, L. M.; Mulvaney, P. Gold nanorods: Synthesis, characterization and applications. Coord. Chem. Rev. 2005, 249, 1870-1901.

(10) Sau, T. K.; Rogach, A. L.; Jäckel, F.; Klar, T. A.; Feldmann, J. Properties and Applications of Colloidal Nonspherical Noble Metal Nanoparticles. Adv. Mater. 2010, 22, 1805-1825.

(11) Link, S.; Mohamed, M. B.; El-Sayed, M. A. Simulation of the Optical Absorption Spectra of Gold Nanorods as a Function of Their Aspect Ratio and the Effect of the Medium Dielectric Constant. J. Phys. Chem. B 1999, 103, 3073-3077.

(12) Katz, E.; Willner, I. Integrated Nanoparticle-Biomolecule Hybrid Systems: Synthesis, Properties, and Applications. Angew. Chem., Int. Ed. 2004, 43, 6042-6108.

(13) Hainfeld, J. F.; Powell, R. D. New Frontiers in Gold Labeling. J. Histochem. Cytochem. 2000, 48, 471-480.

(14) Mason, J. N.; Farmer, H.; Tomlinson, I. D.; Schwartz, J. W.; Savchenko, V.; DeFelice, L. J.; Rosenthal, S. J.; Blakely, R. D. Novel fluorescence-based approaches for the study of biogenic amine transporter localization, activity, and regulation. J. Neurosci. Methods 2005, 143, 3-25.

(15) Hamad-Schifferli, K.; Schwartz, J. J.; Santos, A. T.; Zhang, S.; Jacobson, J. M. Remote electronic control of DNA hybridization through inductive coupling to an attached metal nanocrystal antenna. Nature 2002, 415, 152-155.

(16) Yang, M.; Chung, F. L.; Thompson, M. Acoustic network analysis as a novel technique for studying protein adsorption and denaturation at surfaces. Anal. Chem. 1993, 65, 3713-3716.

(17) Hobara, D.; Niki, K.; Zhou, C.; Chumanov, G.; Cotton, T. M. Characterization of cytochurome $\mathrm{c}$ immobilized on modified gold and silver electrodes by surface-enhanced Raman spectroscopy. Colloids Surf., A 1994, 93, 241-250.

(18) Lofas, S. Dextran modified self-assembled monolayer surfaces for use in biointeraction analysis with surface plasmon resonance. Pure Appl. Chem. 1995, 67, 829-834.

(19) Yu, X.; Lei, D. Y.; Amin, F.; Hartmann, R.; Acuna, G. P.; Guerrero-Martínez, A.; Maier, S. A.; Tinnefeld, P.; Carregal-Romero, S.; Parak, W. J. Distance control in-between plasmonic nanoparticles via biological and polymeric spacers. Nano Today 2013, 8, 480-493.

(20) Malikova, N.; Pastoriza-Santos, I.; Schierhorn, M.; Kotov, N. A.; Liz-Marzán, L. M. Layer-by-Layer Assembled Mixed Spherical and Planar Gold Nanoparticles: Control of Interparticle Interactions. Langmuir 2002, 18, 3694-3697.
(21) Crumbliss, A. L.; Stonehuerner, J. G.; Henkens, R. W.; Zhao, J.; O'Daly, J. P. A carrageenan hydrogel stabilized colloidal gold multienzyme biosensor electrode utilizing immobilized horseradish peroxidase and cholesterol oxidase/cholesterol esterase to detect cholesterol in serum and whole blood. Biosens. Bioelectron. 1993, 8 , 331-337.

(22) Keating, C. D.; Kovaleski, K. M.; Natan, M. J. Protein:Colloid Conjugates for Surface Enhanced Raman Scattering: Stability and Control of Protein Orientation. J. Phys. Chem. B 1998, 102, 94049413.

(23) Austin, L. A.; Mackey, M. A.; Dreaden, E. C.; El-Sayed, M. A. The optical, photothermal, and facile surface chemical properties of gold and silver nanoparticles in biodiagnostics, therapy, and drug delivery. Arch. Toxicol. 2014, 88, 1391-1417.

(24) Huang, X.; Neretina, S.; El-Sayed, M. A. Gold Nanorods: From Synthesis and Properties to Biological and Biomedical Applications. Adv. Mater. 2009, 21, 4880-4910.

(25) Hildebrandt, P.; Stockburger, M. Cytochrome $c$ at charged interfaces. 1. Conformational and redox equilibria at the electrode/ electrolyte interface probed by surface-enhanced resonance Raman spectroscopy. Biochemistry 1989, 28, 6710-6721.

(26) Weidinger, I. M.; Murgida, D. H.; Dong, W.-f.; Möhwald, H.; Hildebrandt, P. Redox Processes of Cytochrome c Immobilized on Solid Supported Polyelectrolyte Multilayers. J. Phys. Chem. B 2006, $110,522-529$.

(27) Murphy, C. J.; Thompson, L. B.; Alkilany, A. M.; Sisco, P. N.; Boulos, S. P.; Sivapalan, S. T.; Yang, J. A.; Chernak, D. J.; Huang, J. The Many Faces of Gold Nanorods. J. Phys. Chem. Lett. 2010, 1, 2867-2875.

(28) Burrows, N. D.; Lin, W.; Hinman, J. G.; Dennison, J. M.; Vartanian, A. M.; Abadeer, N. S.; Grzincic, E. M.; Jacob, L. M.; Li, J.; Murphy, C. J. Surface Chemistry of Gold Nanorods. Langmuir 2016, 32, 9905-9921.

(29) Aubin-Tam, M.-E.; Hamad-Schifferli, K. Structure and function of nanoparticle-protein conjugates. Biomed. Mater. 2008, 3, 034001.

(30) Królikowska, A.; Bukowska, J. Self-assembled monolayers of mercaptosuccinic acid monolayers on silver and gold surfaces designed for protein binding. Part II: vibrational spectroscopy studies on cytochrome c immobilization. J. Raman Spectrosc. 2007, 38, 943-949.

(31) Aubin-Tam, M.-E.; Hamad-Schifferli, K. Gold NanoparticleCytochrome c Complexes: The Effect of Nanoparticle Ligand Charge on Protein Structure. Langmuir 2005, 21, 12080-12084.

(32) Aubin-Tam, M.-E.; Hwang, W.; Hamad-Schifferli, K. Sitedirected nanoparticle labeling of cytochrome c. Proc. Natl. Acad. Sci. U.S.A. 2009, 106, 4095-4100.

(33) Brown, K. R.; Fox, A. P.; Natan, M. J. Morphology-Dependent Electrochemistry of Cytochrome $\mathrm{c}$ at Au Colloid-Modified $\mathrm{SnO}_{2}$ Electrodes. J. Am. Chem. Soc. 1996, 118, 1154-1157.

(34) Park, J.; Yoon, D.-Y.; Kim, Y. Applications of silver nanoplates as colorimetric indicators of $\mathrm{pH}$-induced conformational changes in cytochrome c. Korean J. Chem. Eng. 2009, 26, 258-260.

(35) Jiang, X.; Jiang, J.; Jin, Y.; Wang, E.; Dong, S. Effect of Colloidal Gold Size on the Conformational Changes of Adsorbed Cytochrome c: Probing by Circular Dichroism, UV-Visible, and Infrared Spectroscopy. Biomacromolecules 2005, 6, 46-53.

(36) Basiruddin, S. K.; Saha, A.; Pradhan, N.; Jana, N. R. Functionalized Gold Nanorod Solution via Reverse Micelle Based Polyacrylate Coating. Langmuir 2010, 26, 7475-7481.

(37) Margoliash, E.; Frohwirt, N. Appendix-Spectrum of horse-heart cytochrome c. Biochem. J. 1959, 71, 570-572.

(38) Gong, J.; Yao, P.; Duan, H.; Jiang, M.; Gu, S.; Chunyu, L. Structural Transformation of Cytochrome $\mathrm{c}$ and Apo Cytochrome $\mathrm{c}$ Induced by Sulfonated Polystyrene. Biomacromolecules 2003, 4, 1293 1300.

(39) Messina, R.; Holm, C.; Kremer, K. Polyelectrolyte Multilayering on a Charged Sphere. Langmuir 2003, 19, 4473-4482.

(40) Burke, S. E.; Barrett, C. J. Controlling the physicochemical properties of weak polyelectrolyte multilayer films through acid/base equilibria. Pure Appl. Chem. 2004, 76, 1387-1398. 
(41) Aulich, D.; Hoy, O.; Luzinov, I.; Brücher, M.; Hergenröder, R.; Bittrich, E.; Eichhorn, K.J.; Uhlmann, P.; Stamm, M.; Esser, N.; Hinrichs, K. In Situ Studies on the Switching Behavior of Ultrathin Poly(acrylic acid) Polyelectrolyte Brushes in Different Aqueous Environments. Langmuir 2010, 26, 12926-12932.

(42) Gittins, D. I.; Caruso, F. Tailoring the Polyelectrolyte Coating of Metal Nanoparticles. J. Phys. Chem. B 2001, 105, 6846-6852.

(43) Verma, A.; Rotello, V. M. Surface recognition of biomacromolecules using nanoparticle receptors. Chem. Commun. 2005, 303-312.

(44) Vertegel, A. A.; Siegel, R. W.; Dordick, J. S. Silica Nanoparticle

Size Influences the Structure and Enzymatic Activity of Adsorbed Lysozyme. Langmuir 2004, 20, 6800-6807.

(45) Aubin-Tam, M.-E.; Zhou, H.; Hamad-Schifferli, K. Structure of cytochrome $\mathrm{c}$ at the interface with magnetic $\mathrm{CoFe} 2 \mathrm{O} 4$ nanoparticles. Soft Matter 2008, 4, 554-559.

(46) Laquintana, V.; Denora, N.; Lopalco, A.; Lopedota, A.; Cutrignelli, A.; Lasorsa, F. M.; Agostino, G.; Franco, M. Translocator Protein Ligand-PLGA Conjugated Nanoparticles for 5-Fluorouracil Delivery to Glioma Cancer Cells. Mol. Pharmaceutics 2014, 11, 859871.

(47) Depalo, N.; Carrieri, P.; Comparelli, R.; Striccoli, M.; Agostiano, A.; Bertinetti, L.; Innocenti, C.; Sangregorio, C.; Curri, M. L. Biofunctionalization of Anisotropic Nanocrystalline SemiconductorMagnetic Heterostructures. Langmuir 2011, 27, 6962-6970.

(48) Hu, Y.; Jiang, X.; Ding, Y.; Ge, H.; Yuan, Y.; Yang, C. Synthesis and characterization of chitosan-poly(acrylic acid) nanoparticles. Biomaterials 2002, 23, 3193-3201.

(49) Speare, J. O.; Rush, T. S. IR spectra of cytochrome c denatured with deuterated guanidine hydrochloride show increase in $\beta$ sheet. Biopolymers 2003, 72, 193-204.

(50) Hu, S.; Morris, I. K.; Singh, J. P.; Smith, K. M.; Spiro, T. G. Complete assignment of cytochrome $\mathrm{c}$ resonance Raman spectra via enzymic reconstitution with isotopically labeled hemes. J. Am. Chem. Soc. 1993, 115, 12446-12458.

(51) Hildebrandt, P.; Stockburger, M. Surface-enhanced resonance Raman spectroscopy of cytochrome $\mathrm{c}$ at room and low temperatures. J. Phys. Chem. 1986, 90, 6017-6024.

(52) Jentzen, W.; Ma, J.-G.; Shelnutt, J. A. Conservation of the conformation of the porphyrin macrocycle in hemoproteins. Biophys. J. 1998, 74, 753-763.

(53) Shelnutt, J. A.; Song, X.-Z.; Ma, J.-G.; Jia, S.-L.; Jentzen, W.; Medforth, C. J.; Medforth, C. J. Nonplanar porphyrins and their significance in proteins. Chem. Soc. Rev. 1998, 27, 31-42.

(54) Yeh, S.-R.; Rousseau, D. L. Folding intermediates in cytochrome c. Nat. Struct. Biol. 1998, 5, 222-228.

(55) Döpner, S.; Hildebrandt, P.; Rosell, F. I.; Mauk, A. G. Alkaline Conformational Transitions of Ferricytochrome c Studied by Resonance Raman Spectroscopy. J. Am. Chem. Soc. 1998, 120, $11246-11255$.

(56) Santoni, E.; Scatragli, S.; Sinibaldi, F.; Fiorucci, L.; Santucci, R.; Smulevich, G. A model for the misfolded bis-His intermediate of cytochrome c: the 1-56 N-fragment. J. Inorg. Biochem. 2004, 98, 1067-1077.

(57) Caroppi, P.; Sinibaldi, F.; Santoni, E.; Howes, B. D.; Fiorucci, L.; Ferri, T.; Ascoli, F.; Smulevich, G.; Santucci, R. The 40s $\Omega$-loop plays a critical role in the stability and the alkaline conformational transition of cytochrome c. J. Biol. Inorg Chem. 2004, 9, 997-1006.

(58) Tognaccini, L.; Ciaccio, C.; D’Oria, V.; Cervelli, M.; Howes, B. D.; Coletta, M.; Mariottini, P.; Smulevich, G.; Fiorucci, L. Structurefunction relationships in human cytochrome c: The role of tyrosine 67. J. Inorg. Biochem. 2016, 155, 56-66.

(59) Milazzo, L.; Tognaccini, L.; Howes, B. D.; Sinibaldi, F.; Piro, M. C.; Fittipaldi, M.; Baratto, M. C.; Pogni, R.; Santucci, R.; Smulevich, G. Unravelling the Non-Native Low-Spin State of the Cytochrome cCardiolipin Complex: Evidence of the Formation of a His-Ligated Species Only. Biochemistry 2017, 56, 1887-1898.

(60) Ciaccio, C.; Tognaccini, L.; Battista, T.; Cervelli, M.; Howes, B. D.; Santucci, R.; Coletta, M.; Mariottini, P.; Smulevich, G.; Fiorucci, L. The Met80Ala and Tyr67His/Met80Ala mutants of human cytochrome $\mathrm{c}$ shed light on the reciprocal role of Met80 and Tyr67 in regulating ligand access into the heme pocket. J. Inorg. Biochem. 2017, 169, 86-96.

(61) Milazzo, L.; Tognaccini, L.; Howes, B. D.; Coppola, D.; Smulevich, G. Probing the non-native states of Cytochrome $c$ with resonance Raman spectroscopy: A tool for investigating the structurefunction relationship. J. Raman Spectrosc. 2018, DOI: 10.1002/ jrs.5315.

(62) Placido, T.; Fanizza, E.; Cosma, P.; Striccoli, M.; Curri, M. L.; Comparelli, R.; Agostiano, A. Electroactive Layer-by-Layer Plasmonic Architectures Based on Au Nanorods. Langmuir 2014, 30, 2608-2618.

(63) Khanal, B. P.; Zubarev, E. R. Rings of Nanorods. Angew. Chem., Int. Ed. 2007, 46, 2195-2198.

(64) Placido, T.; Comparelli, R.; Giannici, F.; Cozzoli, P. D.; Capitani, G.; Striccoli, M.; Agostiano, A.; Curri, M. L. Photochemical Synthesis of Water-Soluble Gold Nanorods: The Role of Silver in Assisting Anisotropic Growth. Chem. Mater. 2009, 21, 4192-4202. 